\title{
ON THE SIRS EPIDEMIC MODEL WITH FREE BOUNDARIES
}

\author{
J. O. TAKHIROV and Z. K. DJUMANAZAROVA \\ Institute of Mathematics \\ Uzbek Academy of Science \\ Ulugbek Str., 81 \\ Tashkent, 100125 \\ Uzbekistan \\ e-mail:prof.takhirov@yahoo.com
}

\begin{abstract}
We investigate an epidemic non-linear reaction-diffusion system with two free boundaries. A free boundary is introduced to describe the expanding front of the infectious environment. A priori estimates of the required functions are established, which are necessary for the correctness and global solvability of the problem. We get sufficient conditions for the spread or disappearance of the disease. It has been proven that with a base reproductive number $R_{0} \geq 1$, the disease disappears in the long term if the initial values and the initial area are sufficiently small.
\end{abstract}

\section{Introduction}

It is known that mathematical modelling is an effective approach to studying the spread of infectious diseases, since it can identify the main factors underlying the mechanisms of transmission and provide real 2020 Mathematics Subject Classification: 35K57, 35K61, 92D30.

Keywords and phrases: reaction-diffusion systems, epidemic model, free boundary, asymptotic behaviour of the solution.

Received April 14, 2021

(C) 2021 Scientific Advances Publishers 
strategies for managing the situation. Since the appearance of the first works (see [1], [2]), mathematical epidemiology has undergone extraordinary development. All of this is based on the refinement of several basic models with a simple structure, where the population is divided into states or "compartments" representing status in relation to infection, such as, for example, susceptibility, infection and immunity. These models of the spread of infection are deterministic, continuous in time and expressed by a system of nonlinear ordinary differential equations (ODE). The main dynamic characteristics of these models are the presence of a disease-free equilibrium and, provided that the corresponding parameter reflecting the spread of infection exceeds a threshold, the endemic equilibrium at which the infection persists. The key question concerns the sustainability of these equilibria. The following SIRS model was proposed in [3]:

$$
\left\{\begin{array}{l}
S^{\prime}=b(N-p I)-\mu S-\frac{\beta S I}{N}+\alpha \\
I^{\prime}=b p I+\frac{\beta S I}{N}-(\mu+v+\delta) I \\
R^{\prime}=v I-(\alpha+\mu) R
\end{array}\right.
$$

where $S(t), I(t)$ and $R(t)$ denote the size of susceptible, infected and removed individuals, respectively and $N=S+I+R ; b>0$ and $\mu>0$ are the birth and death rates, $\beta>0$ is the transmission rate, $v>0$ is the rate of recovery from infection, $\delta>0$ is the disease-specific mortality rate, $p \in(0,1)$ is the fraction of vertically infected new born, and $\alpha>0$ is the rate of return to susceptibility by loss of immunity. The stability analysis of the endemic equilibrium was performed by Lyapunov direct method under the very special assumption of constant population size $N$.

In [4], the global stability of equilibrium without diseases is demonstrated and the Lyapunov function is proposed, which allows one to demonstrate the stability of an endemic state in general conditions for (1.1). More complex models are often used today. In order to better 
simulate the spread of the epidemic, the authors of [5] proposed a rich non-linear incidence of $S f(I)$. Since then, other nonlinear incidents have been proposed such as $\beta I^{p} S^{q}, \frac{\beta S I^{p}}{\left(1+\alpha I^{q}\right)}$ and $\frac{\beta I^{p} S}{(1+\alpha S)}$ (see [6]). Research has shown that models of epidemics with non-linear incidence have more complex dynamics than models with bilinear or standard incidence. For example, in [7], Chen built a SIRS model with standard morbidity, disease-related mortality, and transition from infectious to susceptible class.

In [8], the following model of SIRS epidemic with non-linear incidence $S f(I)$ and transition from the infected class is proposed to the class of susceptible

$$
\left\{\begin{array}{l}
\frac{d S}{d t}=\Lambda-\mu S-S f(I)+\gamma_{1} I+\delta R \\
\frac{d I}{d t}=S f(I)-\left(\mu+\gamma_{1}+\gamma_{2}+\alpha\right) I \\
\frac{d R}{d t}=\gamma_{2} I-(\mu+\delta) R
\end{array}\right.
$$

with initial conditions $S(0)=S_{0} \geq 0, I(0)=I_{0} \geq 0, R(0)=R_{0} \geq 0$. Here, $\Lambda$ is the coefficient of replenishment of susceptible individuals, $\mu$ denotes natural mortality, $\gamma_{1}$ is the coefficient of the transition from the infected class to the susceptible class, $\gamma_{2}$ is the coefficient of the transition from the infected class to the recovered class, $\alpha$ is the mortality caused by the disease, and $\delta$ is the coefficient of loss of immunity. It is assumed that $\Lambda$ and $\mu$ are positive, while $\alpha, \gamma_{1}, \gamma_{2}$, and $\delta$ are nonnegative and $f$ satisfies the conditions: (i) $f(0)=0$ and $f(I)>0$ for $I>0$; (ii) $\frac{F(I)}{I}$ is continuous and does not increase monotonically for $I>0$ and $\lim _{I \rightarrow 0}+\frac{f(I)}{I}=\beta>0$. 
In determining potential strategies for combating an infectious disease, the reproduction number $R_{0}$ is the main quantity that determines the intensity of interventions [9]. $R_{0}$ has been effectively used in vaccination epidemiological practice to provide a vaccine coverage threshold for disease eradication. The basic reproduction number $R_{0}$, is the expected number of secondary cases due to one typical infection joining a fully susceptible population during the infectious period [10]. When $R_{0}<1$, the disease will disappear soon. If $R_{0}>1$, the disease will spread among the population and may cause a pandemic. In epidemiology, a next generation matrix is a method used to determine the basic reproduction number $R_{0}$ for a compartment model of infectious disease spread $[11,12]$.

The basic reproduction number $R_{0}$ is usually defined [9] as: the average number of secondary cases caused by a "typical" infected person during his/her life (period of infection). This quantity cannot be calculated explicitly in most cases. Regardless of whether it is possible to calculate $R_{0}$ explicitly, its role in the study of equilibrium stability can still be determined. The most reasonable epidemic models maintain at least two types of equilibrium: disease-free equilibrium and positive (endemic) equilibrium. Usually, it can be shown that a painless equilibrium is locally asymptotically stable if $R_{0}<1$, and unstable if $R_{0}>1$ [13].

We believe that the models described by the ODE system and the dynamic properties of their solutions play an important role in the study of PDE models. The ODE system assumes that all individuals experience the same homogeneous environment. However, in reality, many physical aspects of the environment, such as climate, chemical composition or physical structure, can vary from place to place, and people are randomly distributed in space and tend to interact with the physical environment and other organisms in their spatial neighbourhood. 
In the event of the appearance and reappearance of infectious bacteria, spread begins at the place of their origin and spreads to the places where contact transmission occurs. It is very important and interesting to study how bacteria spread over a large area, causing environmental problems. Based on these considerations, reactiondiffusion equations were then used to study the spread of infectious diseases (see [14, 15]). Huang et al. [16] discussed the dynamics of the reaction-diffusion SIS model as

$$
\begin{gathered}
S_{t}=d_{s} \Delta S-\beta(x) \frac{S I}{S+I}+\gamma(x) I+\Lambda(x), \quad x \in \Omega, \quad t>0, \\
I_{t}=d_{I} \Delta I+\beta(x) \frac{S I}{S+I}-\gamma(x) I, \quad x \in \Omega, \quad t>0,
\end{gathered}
$$

with the Dirichlet boundary condition.

Murray's book [17] contains many examples of solutions for travelling waves and a chapter on the spatial distribution of epidemics. To understand new phenomena of transmission of diseases caused by spatial heterogeneity of the environment, in [18], a model of the epidemiological response SIRS is proposed, which is as follows:

$$
\left\{\begin{array}{l}
S_{t}=\Delta S+\beta-\mu S-\beta S I+\delta R, \quad x \in R, \quad t>0, \\
I_{t}=\Delta I+\beta S I-(\mu+\gamma) I, \quad x \in R, \quad t>0, \\
R_{t}=\Delta R+\gamma I-(\mu+\delta) R, \quad x \in R, \quad t>0,
\end{array}\right.
$$

with initial conditions $S(x, 0)=S_{0}(x), I(x, 0)=I_{0}(x)$ and $R(x, 0)=R_{0}(x)$ for $x \in R$. Just as in [19], denoting $N=S+I+R$, it is found that system (1.3) is equivalent to

$$
\left\{\begin{array}{l}
N_{t}=\Delta N+\beta-\mu N, \quad x \in R, \quad t>0, \\
I_{t}=\Delta I+\beta(N-I-R) I-(\mu+\gamma) I, \quad x \in R, \quad t>0, \\
R_{t}=\Delta R+\gamma I-(\mu+\delta) R, \quad x \in R, \quad t>0,
\end{array}\right.
$$

with initial conditions $N(x, 0)=N_{0}(x), I(x, 0)=I_{0}(x)$, and $R(x, 0)=$ $R_{0}(x)$ for $x \in R$. 
It was shown in [19] that for $R_{0}:=\frac{\beta B}{\mu(\mu+\gamma)}>1$ and $c>c^{*}:=2$ $\sqrt{(\mu+\gamma)\left(R_{0}-1\right)}$, model (1.4) always admits the solution of a travelling wave with speed $c$, linking the disease - free equilibrium $\left(\frac{B}{\mu}, 0,0\right)$ and endemic equilibrium $(N *, I *, R *)$. Biologically, this means that the disease begins and becomes endemic, even if the infected class is limited to a small region at the beginning. This does not correspond to the reality that the disease always gradually rises from a local region and then spreads further into a wider area in terms of spatial distribution.

Recently, problems with a free boundary are often used in the mathematical modelling of various natural processes. In particular, the well-known Stefan condition was used to describe the expansion front in a number of applied problems. For example, it has been used to describe wound healing [20], tumor growth [21], and so on. As a typical example in epidemiology, Kim et al. [22] consider a diffuse epidemic model of SIR in a radically symmetric area with a free boundary, sufficient conditions for the disappearance or spread of the disease are determined by a certain threshold value. In particular, Ge et al. [23] proposed a simplified model of the free boundaries of SIS, they investigated the influence of spatial heterogeneity and advection on the resistance and eradication of an infectious disease. In [24], a free-boundary epidemic model is considered, which describes the front of bacteria spread.

In [25], the dynamics of the spread of the epidemic model is considered, which is modelled by a partially degenerate reactiondiffusion-advection system with free boundaries and a sigmoidal function. The influence of small advection on the dynamics of the spread of the epidemic disease is observed. First, the global existence and uniqueness of the solution is clarified. 
In [26] investigated the epidemic SIRS system with non-linear incidence and free-boundary condition, assuming that the environment is radically symmetric. Besides the main reproductive number, the size of the initial epidemic area and the rate of spread of the disease have also been shown to have important influences on disease transmission.

By discussing the dynamics of the free boundary problem in the SIRS model, the spread of the disease is described. Sufficient conditions have been obtained that ensure the spread or disappearance of the disease. In addition, an estimate of the expansion rate is also given when the free boundaries extend over the whole $R$.

In this work, taking into account the above results, we will try to generalize the constructed models in a certain direction and develop the results obtained.

A model of any system is a copy that reflects the basic characteristics of the system and, thus, can be used to predict the behaviour of the 'original' system under various conditions. Each model has its own assumptions, strengths and limitations, so it is suitable for answering a specific set of questions.

We consider an SIRS epidemic reaction-diffusion nonlinear model with free boundary

$$
\begin{gathered}
N_{t}=\left(d_{1}(N) N_{x}\right)_{x}+b_{1} N_{x}+B-\mu N-c_{1} I \text { in } Q \\
I_{t}=\left(d_{2}(I) I_{x}\right)_{x}+b_{2} I_{x}+c_{2}(N-I-R) I-\left(\mu+\gamma_{1}+\gamma_{2}+c_{1}\right) I \text { in } D \\
R_{t}=\left(d_{3}(R) R_{x}\right)_{x}+b_{3} R_{x}+\gamma_{2} I-(\mu+\delta) R \text { in } D \\
N(0, x)=N_{0}(x),-l \leq x \leq l, I(0, x)=I_{0}(x), R(0, x)=R_{0}(x), \\
\quad-h_{0} \leq x \leq h_{0}, \\
N_{x}(t,-l)=N_{x}(t, l)=0, \quad t \geq 0,
\end{gathered}
$$




$$
\begin{aligned}
& I(t, x)=R(t, x)=0, x=g(t) \text { or } x=h(t), \quad t \geq 0, \\
& g^{\prime}(t)=-\mu_{1} I_{x}(t, g(t)), h^{\prime}(t)=-\mu_{1} I_{x}(t, h(t)), \quad t>0,
\end{aligned}
$$

where

$$
\begin{gathered}
Q=\{(t, x): 0<t,-l<x<l\}, \\
D=\{(t, x): 0<t, g(t)<x<h(t)\}, \\
-l<g(0)=-h_{0}, h(0)=h_{0}<l .
\end{gathered}
$$

We use the changing region $(g(t) ; h(t))$ to denote the infective environment of disease, where the free boundaries $x=g(t)$ and $x=h(t)$ represent the spreading fronts of epidemic and will be determined together with $(N, I, R)$; Here, $B$ is the coefficient of replenishment of susceptible individuals, $\mu$ denotes natural mortality, $\gamma$ is the coefficient of the transition from the infected class to the recovered class, $c_{1}$ denotes the infection rate, $c_{2}$ is the mortality caused by the disease, $\delta$ is the coefficient of loss of immunity. $h_{0}, b, c_{1}, c_{2}, \delta, \mu$ and $\mu_{1}$ are positive constants and the nonlinear diffusion coefficients of biocapacity $d_{i}(\xi)$, $i=1,2,3$ and the positive initial functions $N_{0}(x), I_{0}(x)$, and $R_{0}(x)$ satisfy the following conditions:

(1) $d_{i}(\xi)>d_{i 0}>0, \quad i=1,2,3 \quad d_{i}(\xi) \in C^{1+\alpha}(\Omega)$ for all $\xi$ belonging to a closed set;

(2) $N_{0}(x) \in C^{2}[-l, l], I_{0}(x), R_{0}(x) \in C^{2}\left(\left[-h_{0}, h_{0}\right]\right)$.

Our main goal is to study the influence of advective terms and nonlinear diffusion coefficients on the spread of the epidemic, on the solvability of the problem, and on the asymptotic behaviour of the solution to system (1.5)-(1.11). Unlike others, the problem is considered in a limited area, and the boundary condition (1.9) corresponds to the restrictions on the movement of people. The asymptotic velocities of the front propagation are estimated from below and from above. 
The paper is organized as follows: section "Preliminaries" presents some results for the ODE system related to the definition of reproduction number and stability. Then, step by step, we establish the results required for the global solvability of the problem. Two-sided estimates are established for $N(t, x), I(t, x), R(t, x), g(t), h(t)$, and then $|\cdot|_{1+\alpha},|\cdot|_{2+\alpha}$ estimates for the Holder norms. The uniqueness theorem and the comparison principle are proved, and finally some asymptotic properties of the solution are investigated.

\section{Preliminaries: On the Computation of $\boldsymbol{R}_{0}$}

The importance of contact processes is at the center of the study of threshold phenomena [27]. In their recent book [9] present an extensive and systematic study of threshold phenomena. The following tutorial notes also used the next generation approach for the systematic computation of the basic reproductive number. The examples used come mostly from our own work. Connections between threshold phenomena and stability are also explored. The basic reproductive number $R_{0}$ is typically defined as: the average number of secondary cases produced by a "typical" infected individual during his/her entire life as infectious (infectious period) when introduced in a population of susceptibles. This non-dimensional quantity cannot be computed explicitly in most cases because the mathematical description of what is a "typical" infectious individual is difficult to quantify in populations with high degree of heterogeneity. Regardless of whether or not $R_{0}$ can be computed explicitly, its role on the study of the stability of equilibria can still be determined. Most reasonable epidemic models support at least two type of equilibria: A disease-free equilibria and a positive (endemic) equilibria. Typically, one can show that the disease-free equilibrium is locally asymptotically stable (l.a.s.) if $R_{0}<1$ and unstable whenever $R_{0}>1$. Furthermore, in many examples, it has been shown that $R_{0}>1$ implies the existence of a unique (l.a.s.) endemic equilibrium. Many models found 
in the literature have been used to show that when $R_{0}$ crosses the threshold, $R_{0}=1$, a transcritical bifurcation takes place. That is, asymptotic local stability is transferred from the infectious-free state to the new (emerging) endemic (positive) equilibria. In some situations, it can be shown that the transfer of asymptotic stability is independent of initial conditions, that is, it is global [28].

In this paper, we use the results [8] on the determination of $R_{0}$ for systems of ODEs.

Obviously, every solution of (1.2) with the initial condition exists globally and is nonnegative. Moreover, for such solutions, we have

$$
d(S+I+R) / d t=\Lambda-\mu(S+I+R)-\alpha I \leq \Lambda-\mu(S+I+R),
$$

which implies that the set

$$
\Omega=\left((S, I, R) \in R_{3}+: S+I+R \leq \Lambda_{\mu}\right)
$$

is a positively invariant and attractive set for (1.2). For the global dynamics of (1.2), it suffices to consider (1.2) with initial conditions in $\Omega$.

Model (1.2) always has the disease-free equilibrium $E_{0}=\left(\frac{\Lambda}{\mu}, 0,0\right)$. Moreover, an equilibrium $(S, I, R)$ satisfies the equilibrium equations

$$
\left\{\begin{array}{l}
\Lambda-\mu S-S f(I)+\gamma_{1} I+\delta R=0 \\
S f(I)-\left(\mu+\gamma_{1}+\gamma_{2}+\alpha\right) I=0 \\
\gamma_{2} I-(\mu+\delta) R=0 .
\end{array}\right.
$$

Then one can easily see that an equilibrium must be endemic if it is not disease free. Suppose that $\left(S^{*}, I^{*}, R^{*}\right)$ is an endemic equilibrium. Then

$$
S^{*}=\left(\mu+\gamma_{1}+\gamma_{2}+\alpha\right) I^{*} / f\left(I^{*}\right) \text { and } R^{*}=\frac{\gamma_{2} I^{*}}{(\mu+\delta)} \text {, }
$$

where $I^{*}$ is a positive zero of the function $H$, 
ON THE SIRS EPIDEMIC MODEL WITH FREE BOUNDARIES 31

$$
H(I)=\mu\left(\mu+\gamma_{1}+\gamma_{2}+\alpha\right) I / f(I)+\left(\mu+\alpha+\mu \gamma_{2} /(\mu+\delta)\right) / I-\Lambda .
$$

$H$ is a strictly increasing function on $R+$. Moreover, $\lim _{I \rightarrow 0+} H(I)=\mu\left(\mu+\gamma_{1}\right.$ $\left.+\gamma_{2}+\alpha\right)-\Lambda$ and $H\left(\frac{\Lambda}{\mu}\right)>0$. Therefore, $H$ has a positive zero if and only if $\mu\left(\mu+\gamma_{1} \beta+\gamma_{2}+\alpha\right)-\Lambda<0$ or equivalently $R_{0}=\Lambda \beta / \mu\left(\mu+\gamma_{1}+\gamma_{2}\right.$ $+\alpha)>1$. Thus, the authors established the following results.

Theorem 2.1 (Theorem 1.1, [8]). (i) If $R_{0} \leq 1$, then (1.2) only has the disease-free equilibrium $E_{0}$. (ii) If $R_{0}>1$, besides $E_{0}$, (1.2) also has a unique endemic equilibrium $E^{*}=\left(S^{*}, I^{*}, R^{*}\right)$, where $I^{*}$ is the unique positive zero of $H$ defined by (2.4), and $S^{*}$ and $R^{*}$ are given in (2.3).

Theorem 2.2 (Theorem 2.1, [8]). If $R_{0}<1$, then the disease-free equilibrium $E_{0}$ of (1.2) is asymptotically stable in $\Omega$.

Further, the authors consider the following equivalent system:

$$
\left\{\begin{array}{l}
d I / d t=(N-I-R) f(I)-\left(\mu+\gamma_{1}+\gamma_{2}+\alpha\right) I, \\
d R / d t=\gamma_{2} I-(\mu+\delta) R \\
d N / d t=\Lambda-\mu N-\alpha I .
\end{array}\right.
$$

Notice that $E^{*}=\left(I^{*}, R^{*}, N^{*}\right)$ is the unique endemic equilibrium of (2.5) when $R_{0}>1$.

Theorem 2.3 (Theorem 2.1, [8]). Suppose $R_{0}>1$. Then the endemic equilibrium $E^{*}$ of (2.5) is globally asymptotically stable in $\Omega_{0}=(I, R, N)$ : $I>0, N=S+I+R$. 


\section{Existence and Uniqueness of Solutions}

\subsection{A priori estimates}

Lemma 3.1. Let $(N, I, R, g(t), h(t))$ be solution of problem (1.5)-(1.11) defined for $t \in(0, T]$, where $T \in(0,+\infty)$. Then there exist constants $M_{i}$, $i=\overline{1,4}$ independent of $T$ such that

$$
\begin{gathered}
0<N(t, x) \leq M_{1}=\max \left\{\frac{B}{\mu},\left\|N_{0}\right\|_{\infty}\right\} \text { in } Q_{T}=\{(t, x): 0<t \leq T,-l<x<l\}, \\
0<I(t, x) \leq M_{2}=\max \left\{M_{1},\left\|I_{0}\right\|_{\infty}\right\} \text { in } D_{T}=\{(t, x): 0<t \leq T, g(t)<x<h(t)\}, \\
0<R(t, x) \leq M_{3}=\max \left\{\frac{\gamma_{2}}{\mu+\delta} M_{2},\left\|R_{0}\right\|_{\infty}\right\} \text { in } D_{T}, \\
0<-g^{\prime}(t) \leq M_{1} M_{4}, \quad 0<h^{\prime}(t) \leq M_{1} M_{4} \text { for } 0<t<T,
\end{gathered}
$$

where $M_{4}$ depends on $M_{2}, I_{0}$ and $h_{0}$.

Proof. Using the strong maximum principle to the equations of the system (1.5)-(1.11), we immediately obtain $M_{1} \geq N(t, x)>0$ in $Q$, $I(t, x)>0, R(t, x)>0$ in $D$. Hopf lemma then implies that $I_{x}(t, g(t))>0$, $I_{x}(t, h(t))<0$, for all $t \in(0, T]$. It then follows from the Stefan conditions, that $g^{\prime}(t)<0, h^{\prime}(t)>0, t>0$.

Further arguing as in $[29,30]$ we can obtain the remaining estimates of lemma 3.1.

We will establish Holder norm bounds $|\cdot|_{1+\alpha}$ and $|\cdot|_{2+\alpha}$ in $\bar{Q}_{T}$ and $\bar{D}_{T}$. For each equation of the system, we formulate the corresponding problem

$$
\left\{\begin{array}{l}
\left(d_{1} N_{x}\right)_{x}+a_{1}\left(N, N_{x}, I\right)-N_{t}=0 \quad \text { in } \quad Q_{T} \\
N(0, x)=N_{0}(x), \quad-l \leq x \leq l, \\
N_{x}(t,-l)=N_{x}(t, l)=0, \quad 0 \leq t \leq T,
\end{array}\right.
$$


where $a_{1}\left(N, N_{x}\right)=\left(b_{1} N_{x}+B-\mu N-c_{1} I\right)$,

$$
\left\{\begin{array}{l}
\left(d_{2}(I) I_{x}\right)_{x}+a_{2}\left(I, I_{x}, N, R\right)-I_{t}=0 \quad \text { in } D_{T}, \\
I(0, x)=I_{0}(x), \quad-h \leq x \leq h_{0}, \\
I(t, g(t))=I(t, h(t))=0, \\
g^{\prime}(t)=-\mu_{1} I_{x}(t, g(t)), h^{\prime}(t)=-\mu_{1} I_{x}(t, h(t)),
\end{array}\right.
$$

where $a_{2}\left(I, I_{x}, N, R\right)=\left(b_{2} I_{x}+c_{2}(N-I-R) I-\left(\mu+\gamma_{1}+\gamma_{2}+c_{1}\right) I\right)$,

$$
\left\{\begin{array}{l}
\left(d_{3}(R) R_{x}\right)_{x}+a_{3}\left(R, R_{x}, I\right)-R_{t}=0 \quad \text { in } D_{T} \\
R(0, x)=R_{0}(x) \\
R(t, g(t))=R(t, h(t))=0
\end{array}\right.
$$

where $a_{3}\left(R, R_{x}, I\right)=b_{3} R_{x}+\gamma_{2} I-(\mu+\delta) R$.

Under these assumptions on the initial date, we can assume that $N_{0}(x)=I_{0}(x)=R_{0}(x)=0$.

Theorem 3.1. Assume that $N(t, x), N_{x}(t, x)$ are continuous in $Q_{t}$ and suppose that $N(t, x)$, is a solution for the problem (3.1). Then

$$
\left|N_{x}(t, x)\right| \leq C_{1}\left(M_{1} M_{2}\right) \text { in } \bar{Q}_{T} .
$$

Moreover, if the weak second derivatives $N_{x x}, N_{t x}$ in $L^{2}\left(Q_{T}\right)$, then there exists $\alpha=\alpha\left(M_{1}\right)$, such that

$$
|N|_{1+\alpha, Q_{T}} \leq C_{2}\left(M_{1} C_{1}\right) .
$$

Additionally, assume that, $N(t, x)$ satisfying (3.1) in $\overline{Q_{T}}$, is continuous with its derivatives $u_{t}, u_{x}, u_{x x}$ and $|u|_{2+\alpha, \overline{Q_{T}}}<\infty$. Then

$$
|N|_{2+a, \overline{Q_{T}}} \leq C_{3}\left(M_{1}, C_{1}, C_{2}\right)
$$


Proof. The estimates (3.4)-(3.6) for $(t, x) \in Q_{T}$ are immediate consequences of the results of [31]. For (3.2), a priori estimates are constructed as follows. Interior estimates in $(t, x): 0 \leq t \leq T,-h_{0} \leq x$ $\leq h_{0}$ are established, just like in [31]. In order to get bounds near unknown curves, we perform the transformation

$$
\tau=t, y=\frac{(x-g(t))-((h(t)-x))}{h(t)-g(t)}
$$

and straighten our the boundary. Then $\Omega=(\tau, y): 0<\tau \leq T,-1<y<1$ corresponds to region $D_{T}$, and for the function

$$
u(\tau, y)=I\left(\tau, \frac{1}{2}(y(h-g)+g+h)\right)
$$

we obtain a parabolic problem with bounded coefficients and right-hand side. The rest of the proof is completed by following the steps outlined in [31]. In doing so, we first establish bounds for $\left|R_{x}\right|$ and then for $|R|_{1+\alpha}$. Estimates for the highest derivatives are obtained from the results for linear equations [32].

Now we will prove that the boundaries $x=g(t)$ and $x=h(t)$ do not cross the lateral boundaries $x=-l$ and $x=l$ respectively, which means that the epidemic will not spread beyond considered area.

On the other hand, it is necessary for the correctness of the problem for an arbitrary time value. It follows from Lemma 2.1 that $x=h(t)(x=$ $g(t))$ is monotonic increasing (decreasing) and, therefore, there exists $\left.h_{\infty} \in(0, \infty]\right)\left(g_{\infty} \in[-\infty, 0)\right)$ such that $\lim _{t \rightarrow+\infty} h(t)=h_{\infty}\left(\lim _{t \rightarrow+\infty} g(t)=g_{\infty}\right)$.

Lemma 3.2. Let $(N, I, R, g(t), h(t))$ be the solution of (1.5)-(1.11) and $2 h_{0}+\int_{-h_{0}}^{h_{0}} I_{0}(x) d x<l, c_{2} \frac{B}{\mu} \leq \mu+\gamma_{1}+\gamma_{2}+c_{1}$. Then $0<h(t)-g(t)<l$ and
$h_{\infty}-g_{\infty}<l$. 
Proof. We have

$$
\begin{aligned}
\frac{d}{d t} \int_{g(t)}^{h(t)} I(t, x) d x= & \int_{g(t)}^{h(t)} I_{t}(t, x) d x+I(t, h(t)) h^{\prime}(t)-I(t, g(t)) g^{\prime}(t) \\
= & \int_{g(t)}^{h(t)}\left(d_{1}(I)\right) I_{x x} d x+b_{2} \int_{g(t)}^{h(t)} I_{x}(t, x) d x+\int_{g(t)}^{h(t)}\left[c_{2}(N-I-R) I\right. \\
& \left.-\left(\mu+\gamma_{1}+\gamma_{2}+c_{1}\right) I\right] d x \\
\leq & d_{1}(I) I_{x}(t, h(t))-d_{1}(I) I_{x}(t, g(t)) \\
& +\int_{g(t)}^{h(t)}\left[c_{2} \frac{B}{\mu}-\left(\mu+\gamma_{1}+\gamma_{2}+c_{1}\right) I\right] d x \\
\leq & -\frac{d_{1}(0)}{\mu_{1}} h^{\prime}(t)+\frac{d_{1}(0)}{\mu_{1}} g^{\prime}(t)=\frac{d_{1}(0)}{\mu_{1}}\left(g^{\prime}(t)-h^{\prime}(t)\right) .
\end{aligned}
$$

Integrating from 0 to $t$ gives

$$
h(t)-g(t) \leq 2 h_{0}+\frac{\mu_{1}}{d_{1}(0)} \int_{g_{0}}^{h_{0}} I_{0}(x) d x .
$$

Letting $t \rightarrow \infty$, we have $h_{\infty}-g_{\infty}<l$.

The proof is complete.

\subsection{The uniqueness result}

First we derive an integral expression for the free boundaries. For this, we rewrite the equation for $I(t, x)$ in the form

$$
I_{t}-\left(d_{2}(I) I_{x}\right)_{x}-b_{2} I_{x}=F(N, I, R),
$$

where $F(N, I, R)=c_{2}(N-I-R) I-\left(\mu+\gamma_{1}+c_{1}\right) I$. 
Integrating (3.7) over $D_{t}=\{(\eta, \xi): 0<\eta \leq t, g(\eta)<\xi<h(\eta)\}$, we obtain

$$
\int_{\partial D_{t}}\left(\left(d_{2} I_{\xi}-b_{2} I\right) d \eta+I d \xi\right)=\iint_{D_{t}} F(N, I, R) d \eta d \xi
$$

or

$$
\begin{aligned}
h(t)-g(t)=2 h_{0} & +\frac{\mu_{1}}{d_{1}(0)} \int_{-h_{0}}^{h_{0}} I_{0}((\xi)) d \xi+\frac{\mu_{1}}{d_{1}(0)} \int_{g(t)}^{h(t)} I(t, \xi) d \xi \\
& +\frac{\mu_{1}}{d_{1}(0)} \iint_{D_{t}} F(N, I, R) d \eta d \xi .
\end{aligned}
$$

Theorem 3.2. Suppose that the assumptions of Lemma 2.1 and Theorem 2.1 holds. Then (1.5)-(1.11) has a unique solution.

Proof. Suppose that the functions $N_{i}, I_{i}, R_{i}, g_{i}(t), h_{i}(t), i=1,2$ solve the system (1.5)-(1.11) and $y_{1}(t)=\min \left(g_{1}(t), g_{2}(t)\right), y_{2}(t)=\max$ $\left(g_{1}(t), g_{2}(t)\right), z_{1}(t)=\min \left(h_{1}(t), h_{2}(t)\right), z_{2}(t)=\max \left(h_{1}(t), h_{2}(t)\right)$.

Let us formulate problems for the differences and try to establish some results. For the $N=N_{1}-N_{2}$, we have

$$
\left\{\begin{array}{l}
N_{t}=\left(d_{1}\left(N_{1}\right) N_{x}\right)+N_{2 x} d^{\prime}(\tilde{N}) N_{x}+b_{2} N_{x}-\mu N-c_{1} I, \\
N(0, x)=0, N_{x}(t,-l)=N_{x}(t, l)=0 .
\end{array}\right.
$$

By the maximum principle, we obtain

$$
|N| \leq c_{3} e^{c T} \max _{Q_{t}}|I(t, x)| t,
$$

where $c_{3}$ and $c$ are known constants. 
Taking into account the a priori estimates established above, we find

$$
\begin{gathered}
\left|I_{1}\left(t, y_{2}(t)\right)-I_{2}\left(t, y_{2}(t)\right)\right| \leq K\left|g_{1}(t)-g_{2}(t)\right|, \\
\left|I_{1}\left(t, z_{1}(t)\right)-I_{2}\left(t, z_{1}(t)\right)\right| \leq K\left|h_{1}(t)-h_{2}(t)\right| .
\end{gathered}
$$

Considering the difference $I(t, x)=I_{1}(t, x)-I_{2}(t, x)$, we obtain an equation with bounded and smooth coefficients and the problem

$$
\begin{gathered}
I_{t}=d_{2}(I) I_{x x}+c_{4} I_{x}+c_{5} I+c_{6} N+c_{7} R \text { in } D^{*}, \\
I(0, x)=0, \quad-h_{0} \leq x \leq h_{0}, \\
\left|I\left(t, y_{2}(t)\right) \leq K \max _{0 \leq \eta \leq t}\right| y_{1}(\eta)-y_{2}(\eta) \mid, \\
\left|I\left(t, z_{1}(t)\right) \leq K \max _{0 \leq \eta \leq t}\right| h_{1}(\eta)-h_{2}(\eta) \mid,
\end{gathered}
$$

where $c_{i}, i=4,5,6,7, K$ are known constants, $D^{*}=\{(t, x): 0<t \leq T$, $\left.y_{2}(t)<x<z_{1}(t)\right\}$

From (3.10), using the maximum principle [32], we obtain

$$
\begin{aligned}
|I(t, x)| \leq e^{c t} K\left\{\max _{D^{*}}\left|N(t, x)+\max _{D^{*}}\right| R(t, x) \mid\right. & +\max _{0 \leq \eta \leq t}\left[\left|g_{1}(\eta)-g_{2}(\eta)\right|\right. \\
& \left.\left.+\left|h_{1}(\eta)-h_{2}(\eta)\right|\right]\right\} .
\end{aligned}
$$

A similar (3.9) estimates are established for functions $R(t, x)$.

Considering the difference, we get that

$$
\begin{aligned}
&\left|h_{1}(t)-h_{2}(t)\right|+\left|g_{1}(t)-g_{2}(t)\right| \leq c_{10}\left\{\int_{y_{1}(t)}^{y_{2}(t)}\left|I_{j}(t, \xi)\right| d \xi+\int_{y_{2}(t)}^{z_{1}(t)} \mid I_{1}(t, \xi)\right) \\
&-I_{2}(t, \xi)\left|d \xi+\int_{z_{1}(t)}^{z_{2}(t)}\right| I_{i}(t, \xi)\left|d \xi+\int_{0}^{t} d \eta \int_{y_{1}(\eta)}^{y_{2}(\eta)}\right| F\left(N_{j}, I_{j}, R_{j}\right) \mid d \xi
\end{aligned}
$$




$$
\begin{aligned}
& +\int_{0}^{t} d \eta \int_{y_{2}(\eta)}^{z_{1}(\eta)}\left|F\left(N_{1}, I_{1}, R_{1}\right)-F\left(N_{2}, I_{2}, R_{2}\right)\right| d \xi \\
& \left.+\int_{0}^{t} d \eta \int_{z_{1}(\eta)}^{z_{2}(\eta)}\left|F\left(N_{j}, I_{j}, R_{j}\right)\right| d \xi\right\},
\end{aligned}
$$

where

$$
\begin{aligned}
& \left(N_{j}, I_{j}, R_{j}\right)=\left\{\begin{array}{llc}
N_{1}(t, x), I_{1}(t, x), R_{1}(t, x), & \text { if } & g_{1}(t)<g_{2}(t), \\
N_{2}(t, x), I_{2}(t, x), R_{2}(t, x), & \text { if } & g_{2}(t)<g_{1}(t),
\end{array}\right. \\
& \left(N_{j}, I_{j}, R_{j}\right)=\left\{\begin{array}{llc}
N_{1}(t, x), I_{1}(t, x), R_{1}(t, x), & \text { if } & h_{2}(t)<h_{1}(t), \\
N_{2}(t, x), I_{2}(t, x), R_{2}(t, x), & \text { if } & h_{1}(t)<h_{2}(t),
\end{array}\right.
\end{aligned}
$$

$c_{10}$ - const.

Further, using the ideas and result of [33] the proof of the theorem is completed.

\subsection{The existence result}

It is clear that, the global existence of solutions (1.5)-(1.11) can be derived from local existence using the established a priori estimates for the solutions of the equations and fee boundaries.

Theorem 3.3. There is a $T>0$ such that problem (1.5)-(1.11) admits a unique solution

$$
(N, I, R, g, h) \in C^{1+\alpha, 2+\alpha}\left(Q_{T}\right) \times\left[C^{1+\alpha, 2+\alpha}\left(D_{T}\right)\right]^{2} \times\left[C^{1+\alpha}([0, T])\right]^{2}
$$

satisfying

$$
\begin{aligned}
&\|N\|_{C^{1+\alpha, 2+\alpha}\left(Q_{T}\right)}+\|I\|_{C^{1+\alpha, 2+\alpha}\left(D_{T}\right)}+\|R\|_{C^{1+\alpha, 2+\alpha}\left(D_{T}\right)}+\|h\|_{C^{1+\alpha}([0, T])} \\
&+\|g\|_{C^{1+\alpha}([0, T])} \leq C,
\end{aligned}
$$

where $\alpha \in(0,1), Q_{T}=\{(t, x): 0 \leq t \leq T,-l \leq x \leq l\}, D_{T}=\{(t, x): 0 \leq$ $t \leq T, g(t) \leq x \leq h(t)\}, C$ depend on $h_{0}, \alpha,\left\|N_{0}, I_{0}, R_{0}\right\|_{C^{2}}$. 
Proof. Taking into account the established a priori estimates for all possible solutions and the uniqueness theorem, the Leray-Schauder principle can be applied. The linear problem defines the transformations $w=F(\cdot, k), 0 \leq k \leq 1$, to which the Leray-Schauder principle applies. A fixed point of a operator at $k=1$ is the solution of the problem. In this case, it is easy to check fulfillment of all conditions of the Leray-Schauder principle. A more detailed exposition of the technique can be found, for example, in (Section VII, [34]; Section VI, [32]).

\subsection{The comparison principle}

We exhibit the following comparison principle which can be proven be the similar argument in [35].

Lemma 3.3. Suppose that $T \in(0, \infty), \bar{h}, \bar{g} \in C^{\prime}([0, T]), \bar{N} \in C^{1,2}$ $\left(Q_{T}\right) \cap C\left(\bar{Q}_{T}\right)$ and $\bar{I}, \bar{R} \in C^{1,2}\left(D_{T}^{*}\right) \cap C\left(\bar{D}_{T}^{*}\right)$ with $D_{T}^{*}=\{(t, x): 0<t \leq T$, $\bar{g}(t)<x<\bar{h}(t)\}$, and $\underline{h}, \underline{g} \in C^{1}([0, T]), \underline{N} \in C^{1,2}((0, T] \times(-l, l)) \cap$ $C(0, T] \times[-l, l])$ and $\underline{I}, \underline{R} \in C^{1,2}\left(D_{T}^{* *}\right) \cap C\left(\bar{D}_{T}^{* *}\right)$ with $D_{T}^{* *}=\{(t, x): 0<$ $t \leq T, \underline{g}(t)<x<\underline{h}(t)\}$ satisfying

$$
\begin{aligned}
& \left\{\begin{array}{l}
\bar{N}_{t}-\left(d_{1}(N) \bar{N}_{x}\right)_{x}-b_{1} \bar{N}_{x} \geq B-\mu \bar{N}-c_{1} \underline{I}, \text { in } Q_{T}, \\
\bar{I}_{t}-\left(d_{2}(\bar{I}) \bar{I}_{x}\right)_{x}-b_{2} \bar{I}_{x} \geq c_{2}(\bar{N}-\bar{I}-\underline{R}) \bar{I}-\left(\mu \bar{N}+\gamma_{1}+\gamma_{2}+c_{1}\right) \bar{I}, \quad \text { in } \quad D_{T}^{*}, \\
\bar{R}_{t}-\left(d_{3}(\bar{R}) \bar{R}_{x}\right)_{x}-b_{3} \bar{R}_{x} \geq \gamma_{2} \bar{I}-(\mu+\delta) \bar{R}, \text { in } D_{T}^{*},
\end{array}\right. \\
& \left\{\begin{array}{l}
\underline{N}_{t}-\left(d_{1}(\underline{N}) \underline{N}_{x}\right)_{x}-b_{1} \underline{N}_{x} \geq B-\mu \underline{N}-c_{1} \bar{I}, \text { in } \quad Q_{T}, \\
\underline{I}_{t}-\left(d_{2}(\underline{I}) \underline{I}_{x}\right)_{x}-b_{2} \underline{I}_{x} \geq c_{2}(\underline{N}-\underline{I}-\bar{R}) \underline{I}-\left(\mu+\gamma_{1}+\gamma_{2}+c_{1}\right) \underline{I}, \quad \text { in } \quad D_{T}^{* *}, \\
\underline{R}_{t}-\left(d_{3}(\underline{R}) \underline{R}_{x}\right)_{x}-b_{3} \underline{R}_{x} \geq \gamma_{2} \underline{I}-(\mu+\delta) \underline{R}, \quad \text { in } \quad D_{T}^{* *}, \\
\bar{h}^{\prime}(t) \geqslant-\mu_{1} \bar{I}_{x}(t, \bar{h}(t)), \quad \bar{g}^{\prime}(t) \leq-\mu_{1} \bar{I}_{x}(t, \bar{g}(t)), \quad 0<t \leq T, \\
\underline{h}^{\prime}(t) \leqslant-\mu_{1} \underline{I}_{x}(t, \underline{h}(t)), \quad \underline{g}^{\prime}(t) \geq-\mu_{1} \underline{I}_{x}(t, \underline{g}(t)), \quad 0<t \leq T,
\end{array}\right.
\end{aligned}
$$




$$
\begin{aligned}
& \bar{I}(t, x)=\bar{R}(t, x)=0 \text { for } x \leq \bar{g}(t) \text { and } x \geq \bar{h}(t), \quad 0 \leq x \leq T, \\
& \underline{I}(t, x)=\underline{R}(t, x)=0 \text { for } x \leq \underline{g}(t) \text { and } x \geq \underline{h}(t), \quad 0 \leq x \leq T,
\end{aligned}
$$

$\bar{N}(0, x) \geq N_{0}(x),-l \leq x \leq l, \bar{I}(0, x) \geq I_{0}(x), \bar{R}(0, x) \geq R_{0}(x), x \in\left[-h_{0}, h_{0}\right]$, $\underline{N}(0, x) \leq N_{0}(x),-l \leq x \leq l, \underline{I}(0, x) \leq I_{0}(x), \underline{R}(0, x) \leq R_{0}(x), \underline{g}(0) \leq x \leq \underline{h}(0)$,

$$
\bar{g}(0) \leq h_{0}, \bar{h}(0) \geq h_{0}, \quad \underline{g}(0) \geq-h_{0}, \underline{h}(0) \leq h_{0} .
$$

Then the unique solution ( $N, I, R, g, h)$ of problem (1.5)-(1.11) satisfies

$$
\begin{gathered}
N \leq \bar{N} \text { in } Q_{T}, \quad I \leq \bar{I}, \quad R \leq \bar{R}, \quad g \geq \bar{g} \text { and } h \leq \bar{h} \text { in } D_{T}, \\
N \geq \underline{N} \text { in } Q_{T}, \quad I \geq \underline{I}, \quad R \geq \underline{R}, \quad g \leq \underline{g} \text { and } h \geq \underline{h} \text { for } \underline{g}(t) \leq x \leq \underline{h}(t), \\
0 \leq t \leq T .
\end{gathered}
$$

We can regard $(\bar{N}, \bar{I}, \bar{R}, \bar{g}, \bar{h})$ and $(\underline{N}, \underline{I}, \underline{R}, \underline{g}, \underline{h})$ as the upper and lower solutions of problem (1.5)-(1.11), respectively.

\section{On the Asymptotic Behaviour of the Solutions}

Biologically, $R_{0}$ is called basic reproduction number. We mention that the expression of $R_{0}$ can be also be obtained by applying the next generation matrix method provided by van den Driessche and Watmoung [11]. According to the general result there, $E_{0}$ is locally asymptotically stable if $R_{0}<1$. Regardless of whether or not $R_{0}$ can be computed explicitly, its role on the study of the stability of equilibria can still be determined. Most reasonable epidemic models support at least two type of equilibria and a positive (endemic) equilibria. Li et al. [8] obtained the global dynamics of system (1.2), which is determined by the basic

reproduction number $R_{0}=\frac{B c_{2}}{\mu\left(\mu+\gamma_{1}+\gamma_{2}+c_{1}\right)}$ with LaSalle's invariance principle and the Lyapunov direct method. 
Theorem 4.1. Let $(N, I, R, g, h)$ solution of (1.5)-(1.11) and $R_{0}<1$. Then $\lim _{t \rightarrow \infty}\|N(t, \cdot)\|_{C([-l, l])}=\frac{B}{\mu}, \lim _{t \rightarrow \infty}\|I(t, \cdot)\|_{C([g(t), h(t)])}=0, \lim _{t \rightarrow \infty}\|R(t, \cdot)\|_{C([g(t), h(t)])}=0$ uniformly for all $x \in[g(t), h(t)]$.

Proof. From the comparison principle, $N(t, x) \leq \bar{N}(t)$ for $t \in(0, \infty)$ and $-l \leq x \leq l$, where

$$
\bar{N}(t)=\frac{B}{\mu}=\left(\left\|N_{0}\right\|_{\infty}-\frac{B}{\mu}\right) e^{-\mu t}
$$

which is a solution of

$$
\bar{N}_{t}(t)=B-\mu \bar{N}, \quad t>0, \quad \bar{N}(0)=\left\|N_{0}\right\|_{\infty} .
$$

Since $\lim _{t \rightarrow \infty} \bar{N}(t)=\frac{B}{\mu}$, it follows that $\lim _{t \rightarrow \infty} \bar{N}(t) \leq \frac{B}{\mu}$ for $[-l \leq x \leq l]$.

If $R_{0}<1$, there exists $T_{0}$ such that $\lim _{t \rightarrow \infty} \bar{N}(t) \leq \frac{B}{\mu} \frac{1+R_{0}}{2 R_{0}}$ in $\left[T_{0}, \infty\right) \times-l \leq x \leq l$ we find that $I(t, x)$ satisfies

$$
\left\{\begin{aligned}
& I_{t}=\left(d_{2}(I) I_{x}\right)+b_{2} I_{x} \leq {\left[c_{2} \frac{B}{\mu} \frac{1+R_{0}}{2 R_{0}}-\left(\mu+\gamma_{1}+\gamma_{2}+c_{1}\right)\right] I, } \\
& t>T_{0}, \quad g(t)<x<h(t), \\
& I(g(t), x)=I(h(t), x)=0, \quad t>T_{0} \\
& I\left(T_{0}, x\right)>0, \quad g\left(T_{0}\right)<x<h\left(T_{0}\right) .
\end{aligned}\right.
$$

Since $\frac{c_{2} B}{\mu\left(\mu+\gamma_{1}+\gamma_{2}+c_{1}\right)} \frac{1+R_{0}}{2 R_{0}}<1$, we have $\lim _{t \rightarrow \infty}\|I(t, \cdot)\|_{C([g(t), h(t)])}=0$ and $\lim _{t \rightarrow \infty}\|R(t, \cdot)\|_{C([g(t) h(t)])}=0$ uniformly for all $x \in[g(t), h(t)]$.

The proof is complete. 
Theorem 4.2. Let $R_{0}=1$. Then $h_{\infty}<l$ and $-g_{\infty}<l$ provided that

$$
\mu_{1} \leq \mu_{1}^{*}=\frac{1}{8 M^{*}}
$$

and

$h_{0} \leq h_{*}= \begin{cases}\min \left\{\frac{-2 b_{2}+\sqrt{b_{2}^{2}+d_{20} K}}{4 K}, \frac{-2 b_{3}+\sqrt{b_{3}^{2}+d_{30} \gamma_{2}}}{4 \gamma_{2}}\right\}, \quad K>0, \\ \min \left\{\frac{d_{20}}{8 b_{2}},\right. & \left.\frac{-2 b_{3}+\sqrt{b_{3}^{2}+d_{30} \gamma_{2}}}{4 \gamma_{2}}\right\}, \quad K=0,\end{cases}$

where $K=c_{2} M_{1}-\left(\mu+\gamma_{1}+\gamma_{2}+c_{1}\right), M_{1}=\max \left\{\frac{B}{\mu},\left\|N_{0}\right\|_{\infty}\right\}, M^{*}=\frac{3}{4}$ $\max \left\{\left\|I_{0}\right\|_{\infty},\left\|R_{0}\right\| \infty\right\}$

Proof. As in [18], we take

$$
\begin{gathered}
s(t)=2 h_{0}\left(2-e^{-\gamma t}\right), \quad z(t)=-s(t), t \geq 0, \\
\bar{N}(t, x)=M_{1}, \quad V(y)=1-y^{2}, \quad-1 \leq y \leq 1, \\
\bar{I}(t, x)=\bar{R}(t, x)= \begin{cases}M^{*} e^{-\gamma t} V\left(\frac{x}{s(t)}\right), & z(t) \leq x \leq s(t), \\
0, & x>s(t) \text { or } x<z(t),\end{cases}
\end{gathered}
$$

where $M_{1}=\max \left\{\frac{B}{\mu},\left\|N_{0}\right\|_{\infty}\right\}, \gamma$ and $M^{*}$ are positive constants which will be chosen later. We have

$$
s^{\prime}(t)=2 h_{0} \gamma e^{-\gamma t}, \quad s^{2}(t)=4 h_{0}^{2}\left(2-e^{-\gamma t}\right)^{2}, \quad 0 \leq V \leq 1, \quad y V^{\prime}(y)<0 .
$$

Let $K=C_{2} M_{1}-(\mu+C), C=\left(\gamma_{1}+\gamma_{2}+c_{1}\right)$. Since $R_{0}=1$ we have $K \geq 0$. We consider two cases: $K>0$ and $K=0$. If $K>0$, it is obvious that $\bar{N}_{t}-\left(d_{1}(\bar{N}) \bar{N}_{x}\right)_{x}-b_{2} \bar{N}_{x}=0 \geq B-\mu \bar{N}$, 


$$
\begin{aligned}
& \bar{I}_{t}-\left(d_{2}(\bar{I}) \bar{I}_{x}\right)-b_{2} \bar{I}_{x}-c_{2}(\bar{N}-\bar{I}) \bar{I}+(\mu+x) \bar{I} \\
& \geq \bar{I}_{t}-\left(d_{2} I_{x}\right)_{x}-b_{2} I_{x}-c_{2} \overline{N I}+(\mu+c) \bar{I} \\
& =M^{*} e^{-\gamma t}\left[-\gamma V-\frac{x s^{\prime}(t)}{s^{2}} V^{\prime}-\frac{d_{2}(\bar{I})}{s^{2}(t)} V^{\prime \prime}\right. \\
& \left.-d_{2}^{\prime}(\bar{I}) M^{*} e^{-\gamma t} \frac{\left(V^{\prime}\right)^{2}}{s^{2}}-b_{2} \frac{V^{\prime}}{s(t)}-c_{2} M_{1} V+(\mu+c) V\right] \\
& \geq M^{*} e^{-\gamma t}\left(-\gamma+\frac{d_{20}}{8 h_{0}^{2}}-\frac{b_{2}}{h_{0}}-K\right) \geq 0
\end{aligned}
$$

provided $\gamma=\frac{d_{20}}{16 h_{0}^{2}}, h_{0} \leq \frac{-2 b_{2}+\sqrt{b_{2}^{2}+d_{20} K}}{4 K}$.

Next we find

$$
\begin{aligned}
\bar{R}_{t}-\left(d_{3} \bar{R}_{x}\right)_{x}-b_{3} \bar{R}_{x}-\gamma_{2} \bar{I}_{t}(\mu+\delta) \bar{R} \\
\geq M^{*} e^{-\gamma t}\left(-\gamma+\frac{d_{30}}{8 h_{0}^{2}}-\frac{b_{3}}{h_{0}}-\gamma_{2}\right) \geq 0,
\end{aligned}
$$

if $\gamma=\frac{d_{30}}{16 h_{0}^{2}}$, and $h_{0} \leq \frac{-2 b_{3}+\sqrt{b_{3}^{2}+d_{30} \gamma_{2}}}{4 \gamma_{2}}$.

Now, as in [18] applying the comparison principle, the following inequalities are established: $g(t) \geq z(t)$ and $h(t) \leq f(t)$ for $t>0$. Therefore, an immediately result is $h_{\infty},-g_{\infty}<\lim _{t \rightarrow \infty} s(t)=4 h_{0}<l$. The case $K=0$ is investigated similarly. This completes the proof of the Theorem 4.2.

Theorem 4.2 show that at $R_{0}=1$ the disease disappears in the long term if the initial values and the initial area are small enough. It is known that $R_{0}>1$ means that each infected person is in contact with 
more than one susceptible person at a time, which leads to a higher growth of the epidemic. However, we can find that if $h_{0}$ and $\mu_{1}$ are small enough, the diseases will disappear even if $R_{0}>1$.

Theorem 4.3. If $R_{0}>1$, then $h_{\infty}<l$ and $-g_{\infty}<l$ provided that

$\mu_{1} \leq \mu_{1}^{*}$ and $h_{0} \leq h_{*}^{0}=\left\{\min \left\{\frac{-2 b_{2}+\sqrt{b_{2}^{2}+d_{20} K}}{4 K}, \frac{-2 b_{3}+\sqrt{b_{3}^{2}+d_{30} \gamma_{2}}}{4 \gamma_{2}}\right\}\right.$,

where $\mu_{1}^{*}$ and $K$ are defined in Theorem 4.2.

Proof. Since $R_{0}>1$, the proof is similar to the case $K>0$ in Theorem 4.2. We use Lemma 3.6 and the upper solution constructed here is the same as in Theorem 4.2 .

\section{References}

[1] V. Capasso, Mathematical Structures of Epidemic Systems, Lectures Notes in Biomathematics 97, 2nd Edition, Springer-Verlag, 2008.

[2] W. O. Kermack and A. G. McKendrick, A contribution to the mathematical theory of epidemics, Proceedings of the Royal Society A: Mathematical, Physical, and Engineering Sciences 115(772) (1927), 700-721.

DOI: https://doi.org/10.1098/rspa.1927.0118

[3] S. M. O’Regan, Thomas C. Kelly, Andrei Korobeinikov, Michael J. A. O'Callaghan and Alexei V. Pokrovskii, Lyapunov functions for SIR and SIRS epidemic models, Applied Mathematics Letters 23(4) (2010), 446-448.

DOI: https://doi.org/10.1016/j.aml.2009.11.014

[4] Alberto d'Onofri, Piero Manfredi and Ernesto Salinelli, Lyapunov stability of an SIRS epidemic model with varying population, Arxiv:1812.10676v1 [math.DS]27 Dec 2018.

[5] V. Capasso and G. Serio, A generalization of the Kermack-McKendrick deterministic epidemic model, Mathematical Biosciences 42(1-2) (1978), 43-61.

DOI: https://doi.org/10.1016/0025-5564(78)90006-8

[6] J. Chen, An SIRS epidemic model, Applied Mathematics: A Journal of Chinese Universities 19(1) (2004), 101-108.

DOI: https://doi.org/10.1007/s11766-004-0027-8 
[7] J. Li, Y. Yang, Y. Xiao and S. Liu, A class of Lyapunov functions and the global stability of some epidemic models with nonlinear incidence, Journal of Applied Analysis and Computation 6(1) (2016), 38-46.

DOI: https://doi.org/10.11948/2016004

[8] Ting Li, Fengqin Zhang, Hanwu Liu and Yuming Chen, Threshold dynamics of an SIRS model with nonlinear incidence rate and transfer from infectious to susceptible, Applied Mathematics Letters 70 (2017), 52-57.

DOI: http://dx.doi.org/10.1016/j.aml.2017.03.005

[9] O. Diekmann and J. A. Heesterbeek, Mathematical Epidemiology of Infectious Diseases: Model Building, Analysis and Interpretation, John Wiley and Sons, Chichester, 2000.

[10] J. M. Heffernan, R. J. Smith and L. M. Wahl, Perspectives on the basic reproductive ratio, Journal of the Royal Society Interface 2(4) (2005), 281-293.

DOI: https://doi.org/10.1098/rsif.2005.0042

[11] P. van den Driessche and J. Watmough, Reproduction numbers and sub-threshold endemic equilibria for compartmental models of disease transmission, Mathematical Biosciences 180(1-2) (2002), 29-48.

DOI: https://doi.org/10.1016/S0025-5564(02)00108-6

[12] O. Diekmann, J. A. P. Heesterbeek and M. G. Roberts, The construction of nextgeneration matrices for compartmental epidemic models, Journal of the Royal Society Interface 7(47) (2009), 873-885.

DOI: https://doi.org/10.1098/rsif.2009.0386

[13] P. van den Driessche, Reproduction numbers of infectious disease models, Infectious Disease Modelling 2(3) (2017), 288-303.

DOI: https://doi.org/10.1016/j.idm.2017.06.002

[14] A. Ducrot, M. Langlais and P. Magal, Qualitative analysis and travelling wave solutions for the SI model with vertical transmission, Communications on Pure and Applied Analysis 11(1) (2012), 97-113.

DOI: https://doi.org/10.3934/cpaa.2012.11.97

[15] A. Ducrot and P. Magal, Travelling wave solutions for an infection-age structured epidemic model with external supplies, Nonlinearity 24(10) (2011), 2891-2911.

DOI: https://doi.org/10.1088/0951-7715/24/10/012

[16] W. Z. Huang, M. Han and K. Liu, Dynamics of an SIS reaction-diffusion epidemic model for disease transmission, Mathematical Biosciences \& Engineering 7(1) (2011), 51-66.

DOI: https://doi.org/10.3934/mbe.2010.7.51 
[17] J. D. Murray, Mathematical Biology II: Spatial Models and Biomedical Applications, 3rd Edition, Springer, New York, 2003.

DOI: https://doi.org/10.1007/b98869

[18] J. F. Cao, Wan-Tong Li, Jie Wang and Fei-Ying Yang, A free boundary problem of a diffusive SIRS model with nonlinear incidence, Zeitschrift für Angewandte Mathematik und Physik 68(2) (2017); Article 39.

DOI: https://doi.org/10.1007/s00033-017-0786-8

[19] Q. Gan, R. Xu and P. Yang, Travelling waves of a delayed SIRS epidemic model with spatial diffusion, Nonlinear Analysis: Real World Applications 12(1) (2011), 52-68.

DOI: https://doi.org/10.1016/j.nonrwa.2010.05.035

[20] X. Chen and A. Friedman, A free boundary problem arising in a model of wound healing, SIAM Journal on Mathematical Analysis 32(4) (2000), 778-800.

DPO: https://doi.org/10.1137/S0036141099351693

[21] X. Chen and A. Friedman, A free boundary problem for an elliptic-hyperbolic system: An application to tumor growth, SIAM Journal on Mathematical Analysis 35(4) (2003), 974-986.

DOI: https://doi.org/10.1137/S0036141002418388

[22] K. Kim, Z. Lin and Q. Zhang, An SIR epidemic model with free boundary, Nonlinear Analysis: Real World Applications 14(5) (2013), 1992-2001.

DOI: https://doi.org/10.1016/j.nonrwa.2013.02.003

[23] J. Ge, K. Kim, Z. Lin and H. Zhu, A SIS reaction-diffusion-advection model in a low-risk and high-risk domain, Journal of Differential Equations 259(10) (2015), 5486-5509.

DOI: https://doi.org/10.1016/j.jde.2015.06.035

[24] Inkyung Ahn, Seunghyeon Baek and Zhigui Lin, The spreading fronts of an infective environment in a man-environment-man epidemic model, Applied Mathematical Modelling 40(15-16) (2016), 7082-7101.

DOI: https://doi.org/10.1016/j.apm.2016.02.038

[25] M. Zhao, Wan-Tong Li and Yang Zhang, Dynamics of an epidemic model with advection and free boundaries, Mathematical Biosciences and Engineering 16(5) (2019), 5991-6014.

DOI: https://doi.org/10.3934/mbe.2019300

[26] Kehua Li, Jiemei Li and Wei Wang, Epidemic reaction-diffusion systems with two types of boundary conditions, Electronic Journal of Differential Equations 2018(170) (2018), 1-21. 
[27] Carlos Castillo-Chavez, Jorge X. Velasco-Hernandez and Samuel Fridman, Modelling Contact Structures in Biology, In: Frontiers of Theoretical Biology, Lecture Notes in Biomathematics, Spring-Verlag, New York, 1994.

DOI: https://doi.org/10.1007/978-3-642-50124-1_27

[28] Z. Feng, C. Castilla-Chavez and A. Capurro, A model for Tuberculosis with exogenous reinfection, Theoretical Population Biology 57(3) (2000), 235-247.

DOI: https://doi.org/10.1006/tpbi.2000.1451

[29] C. Tian and S. Ruan, A free boundary problem of Aedes aegypti mosquito invasion, Applied Mathematical Modelling 46 (2017), 203-217.

DOI: https://doi.org/10.1016/j.apm.2017.01.050

[30] J. O. Takhirov, A free boundary problem for a reaction-diffusion equation appearing in biology, Indian Journal of Pure and Applied Mathematics 50(1) (2019), 95-112.

DOI: https://doi.org/10.1007/s13226-019-0309-8

[31] S. N. Kruzhkov, Nonlinear parabolic equations with two independent variable, Transaction of the Moscow Mathematical Society 16 (1967), 329-346.

[32] O. A. Ladyzhenskaja, V. A. Solonnikov and N. N. Ural'ceva, Linear and Quasi-linear Equations of Parabolic Type, American Mathematical Society: Translations of Mathematical Monographs, Vol. 23, Providence, RI, 1968.

DOI: https://doi.org/10.1090/mmono/023

[33] Zh. O. Takhirov and M. S. Rasulov, Problem with free boundary for systems of equations of reaction-diffusion type, Ukrainian Mathematical Journal 69(12) (2018), 1968-1980.

DOI: https://doi.org/10.1007/s11253-018-1481-4

[34] A. Friedman, Partial Differential Equations of Parabolic Type, Courier Dover Publications, 2008.

[35] Y. Du and Zh. Lin, The diffusive competition model with a free boundary: Invasion of a superior or inferior competitor, Discrete and Continuous Dynamical Systems: Series B 19(10) (2014), 3105-3132.

DOI: https://doi.org/10.3934/DCDSB.2014.19.3105 